\title{
The effects of sand surface training on changes in the muscle activity of the paretic side lower limb and the improvement of dynamic stability and gait endurance in stroke patients
}

\author{
Byoung-Ha Hwang, Tae-Ho Kim* \\ Department of Physical Therapy, College of Rehabilitation Science, Daegu University, Gyeongsan, Korea
}

Subjects were divided into two groups, for sand or general surface training. The gait abilities of the patients were evaluated with the Timed Up and Go (TUG) tests and the 6-min walk test (6MWT). Muscle activity in the lower limbs was evaluated with surface electromyography obtained during general surface gait. Gait ability and muscle activity were re-evaluated after gait training for 6 weeks on a sand or general surface. The TUG test was performed 3 times, and an average of the three values was used for analysis. 6MWT was measured once under maximum effort. Electromyography was evaluated using average gait cycle values. In the TUG test, performance times decreased in both groups, while in the 6MWT, performance distance significantly increased only in the sand surface training group. Results from surface electromyography showed that activity of the rectus femoris, biceps femoris, and tibi- alis anterior muscles of the paretic side lower limb significantly decreased after the both interventions. When environments are similar, gait training is helpful for increasing dynamic stability; however, repeated training does not significantly improve gait endurance. Training on a continuously unstable bearing surface such as sand can improve gait endurance by utilizing diverse muscle groups and increasing joint motility. Sand surface training recruited the activity of more muscle groups than did general surface training, and sand-trained participants showed longer gait distances with lower muscle activity levels when they resumed general training than their general-surface-trained counterparts.

Keywords: Hemiplegia, Stroke, Gait training, Sand surface

\section{INTRODUCTION}

Stroke patients have difficulty with normal postural control and reduced gait abilities due to muscle weakening, changes in muscle tone, and damage to the sensory system (Bonan et al., 2004). In particular, reductions in gait velocity and endurance and the loss of dynamic balance ability increase the patient's risk and fear of falling, leading to further difficulties in postural control and reduced social participation (Schmid et al., 2012). Most stroke patients report reduced quality of life even when they have recovered these functions due to a sense of psychological and social loss (Raju et al., 2010). Gait training is thereby an extremely important element in a stroke patient's recovery.
Enabling stroke patients to move independently significantly improves their activity levels. Understanding the relationship between the physical environment and the ability to move at different stages of treatment is particularly important (Patla and Shumway-Cook, 1999). Several studies on how to best enhance stroke patients' gait abilities have been conducted. However, most of these studies have trained patients on a treadmill (Franceschini et al., 2009) or strengthened their muscles using expensive isokinetic exercise equipment (Maynard et al., 2005). These previous studies were conducted in environments that differed from the actual gait environments of the patients, and improvements in gait ability in indoor environments were not recapitulated in outdoor environments (Lord et al., 2008). This break down occurred because di-
${ }^{*}$ Corresponding author: Tae-Ho Kim (iD https://orcid.org/0000-0001-7939-2139 Department of Physical Therapy, College of Rehabilitation Science, Daegu University, 201 Daegudae-ro, Jillyang-eup, Gyeongsan 38453, Korea E-mail: ptkimth@daegu.ac.kr

Received: March 28, 2019 / Accepted: May 27, 2019
This is an Open Access article distributed under the terms of the Creative Commons Attribution Non-Commercial License (http://creativecommons.org/licenses/by-nc/4.0/) which permits unrestricted non-commercial use, distribution, and reproduction in any medium, provided the original work is properly cited. 
verse tasks must be performed to cope with changes in natural environments, and these can be complicated to simulate (ShumwayCook et al., 2002).

To enable stroke patients to walk properly in the real-life environments they will encounter upon returning to their communities, feet function is crucial, as the feet are the first to come into contact with the ground. Efficient gaits require center of gravity motion, stability, impact absorption, and weight support through range of motion provided by strong joints and muscles (Neumann, 2013). It has been reported that feet and ankle functions are improved through training on unstable bearing surfaces, which enhance muscle strength and balance ability (Park et al., 2013); ankle joints as well as knee joints are more stabilized, and motor control is improved (Caraffa et al., 1996; Carter et al., 2006).

In the present study, sand was used as an unstable surface environment for gait training. Sand surface walking requires the motility of various joints, induces diverse angular speeds, and is used by many athletes when training to improve their athletic performance. However, few studies have examined stroke patient gait training on sand surfaces. As such, the present study examined the effects of gait training on sand surfaces for the improvement of dynamic balance ability and gait endurance in stroke patients as well as the changes that occur in the muscle activity of the paretic leg according to different gait training surfaces.

\section{MATERIALS AND METHODS}

\section{Patients}

Out of a total of 30 patients who initially enrolled in the study, two were discharged from the hospital before the study was completed, leaving 28 patients who participated in the full slate of treatments. The experimental group contained 14 patients representing eight cases of right hemiplegia and six cases of left hemiplegia. Group characteristics were six males and eight females, with a mean age of $69.00 \pm 10.0$ years, a mean weight of $61.50 \pm$ $8.1 \mathrm{~kg}$, a mean height of $162.43 \pm 5.7 \mathrm{~cm}$, and a mean time after onset of stroke of $21.00 \pm 13.7$ months. The control group contained 14 patients representing seven cases of right hemiplegia and seven cases of left hemiplegia. Group characteristics were five males and nine females with a mean age of $71.27 \pm 10.5$ years, a mean weight of $65.93 \pm 15.3 \mathrm{~kg}$, a mean height of $161.80 \pm 7.7 \mathrm{~cm}$, and a mean time after onset of stroke of $32.13 \pm 10.5$ months. There were no significant differences in baseline parameters between the experimental and control groups $(P \geq 0.05)$.

The purpose of the present study was explained to the chronic stroke patients who had been hospitalized and were undergoing in-patient treatment. Patients that volunteered to participate in the study were randomly assigned to either the experimental or control group. Subjects were selected from among patients who could walk independently with or without walking aids, had no other musculoskeletal system diseases or surgeries, and had no cognition problems. The Daegu University Institutional Review Board (1040621-201507-HR-003-02) approved all study procedures.

\section{Surface EMG recording and data processing}

Electromyographic data were obtained with a TeleMyo DTS (Noraxon Inc., Scottsdale, AZ, USA) on the rectus femoris, biceps femoris, tibialis anterior, and gastrocnemius muscles, all of which contribute to gait ability, to measure changes in muscle activity in the lower limbs of each patient for the different intervention methods. The electrode of the surface electromyograph for the rectus femoris was attached at a point $5 \mathrm{~cm}$ above the superior margin of the patella; that for the biceps femoris was attached to a point lateral to the midpoint on the line between the popliteal fossa crease and the gluteal crease; that for the tibialis anterior was attached to a point $2 \mathrm{~cm}$ distally and laterally from the line of the anterior tibia; and that for the gastrocnemius was attached laterally to a point $2 \mathrm{~cm}$ below the center line of the popliteal fossa (Schulthies et al., 1998). The earth electrode used in the study was a disposable dual surface electrode made of $\mathrm{Ag}$ and $\mathrm{AgCl}$.

Electrode attachment regions were shaved, cleaned 3 times with alcohol swabs, and allowed to dry before electrodes were attached. In the case of the nonparetic side, maximum contraction was induced in individual muscles to attach electrodes where muscle contraction was the most clearly visible by the naked eye. In the case of the paretic side where maximum voluntary contraction was impossible due to hemiplegia, the patients were instructed to take an upright standing posture so that the electrodes could be attached to the surfaces of the paretic-side muscles in the same positions as on the nonparetic side muscles.

Since voluntary isometric contraction on the paretic side is difficult for hemiplegic patients, participants were asked to maintain a standing posture for $10 \mathrm{sec}$, and the value during the middle 3 sec was used as the maximum isometric contraction reference value. All collected electromyogram signals were subjected to filtering and another signal processing. The electromyogram signal sampling rate was set to $1,000 \mathrm{~Hz}$. Amplified waveforms were filtered through a $40-400 \mathrm{~Hz}$ band pass filter and $60-\mathrm{Hz}$ notch filters to remove noise; signals were then quantified using root mean 
square calculations. Signals collected from the individual muscles during gait were normalized as a percentage of the voluntary reference contraction values measured from the standing posture.

\section{Experimental procedure}

The gait abilities of both groups were evaluated 3 days before the intervention using the 6-min walk test (6MWT) and the Timed Up and Go (TUG) test. These tests were conducted on different days so that one would not be affected by the other. Muscle activities were measured through surface electromyography while patients were walking on generalized surfaces to collect relevant data and to measure the muscle activity of both legs during gait. Both groups underwent gait training for 30 min twice a day, 5 days per week for 6 weeks on either unstable or stable surfaces. Upon completion of the intervention regimen, 6MWT and TUG tests were repeated, and surface electromyography values were measured under the same conditions as the initial tests. Obtained data were then analyzed.

The sand surface training group underwent gait training in bare feet on a specially prepared surface that was $1 \mathrm{~m}$ wide, $5 \mathrm{~m}$ long, and $30 \mathrm{~cm}$ deep. The general surface training group underwent gait training in the treatment room, wearing comfortable shoes, walking along a provided line. TUG tests were conducted three times in total, and values were averaged for use in data analysis. The TUG test is used to evaluate dynamic balance ability and gait velocity and has been verified for intrarater reliability and interrater reliability (Podsiadlo and Richardson, 1991).

The 6MWT was conducted only once because of its challenges to the patients. Each patient went as far as he or she could go, and in cases where the patient gave up or had difficulties, the test was immediately stopped, and the distance walked in that time was

Table 1. Comparison of walking ability evaluation before and after the intervention

\begin{tabular}{lcccc}
\hline Group & Pretest & Posttest & $Z$ & $P$-value \\
\hline TUG & & & & \\
G $(n=14)$ & $30.89 \pm 18.55$ & $27.67 \pm 18.24$ & -3.296 & $0.001^{*}$ \\
SG $(n=14)$ & $27.64 \pm 15.72$ & $21.53 \pm 13.22$ & -3.296 & $0.001^{*}$ \\
$Z$ & & -1.655 & & \\
6MWT & & & & \\
G $(n=14)$ & $159.50 \pm 124.02$ & $182.71 \pm 131.14$ & -1.852 & 0.064 \\
SG $(n=14)$ & $162.00 \pm 148.93$ & $204.93 \pm 158.31$ & -2.669 & $0.008^{*}$ \\
$Z$ & & -1.541 & & \\
\hline
\end{tabular}

Values are presented as mean \pm standard deviation.

G, ground gait training group; SG, sand gait training group; TUG, Timed Up and Go test; 6MWT, 6-min walking test.

${ }^{*} P<0.05$. measured and used for data analysis. This test is also known to be a highly reliable and valid gait endurance evaluation tool (Mossberg and Fortini, 2012).

\section{Statistical analyses}

Kolmogorov-Smirnov tests were conducted to test the normality of the measured data. Independent $t$-tests were conducted to compare average values between the sand and general surface gait groups. Paired $t$-tests were conducted to compare values obtained before and after the intervention. Differences between the two groups before and after the intervention were tested ex post facto using Mann-Whitney $U$-tests. Statistical analyses were conducted with IBM SPSS ver. 18.0 (IBM Co., Armonk, NY, USA), and the statistical significance threshold was set at 0.05 . Data deemed to have errors resulting from individual patient characteristics were excluded from statistical analyses.

\section{RESULTS}

Both groups showed significant increases after the intervention. While there were no differences between groups in final TUG tests for gait ability, only the sand surface training group showed significant increases in performance distance in the 6MWT (Table 1). According to electromyography data obtained on a generalized surface after the interventions, the general surface gait training group showed significant decreases in the muscle activity of the paretic side biceps femoris muscle, while the sand surface training group showed significant decreases in the muscle activity of the rectus femoris and tibialis anterior muscles (Table 2).

Table 2. The comparison of paretic side lower extremity muscle different in gait between gait and san gait group (unit: \%RVC)

\begin{tabular}{ccccc}
\hline Group & Pretest & Posttest & Different & $P$-value \\
\hline G (n=12) & & & & \\
G.HB.M & $134.37 \pm 56.65$ & $102.55 \pm 61.31$ & $31.82 \pm 9.85$ & $0.008^{*}$ \\
G.HB.P & $319.13 \pm 169.39$ & $216.37 \pm 180.97$ & $102.76 \pm 26.66$ & $0.003^{*}$ \\
SG $(n=10)$ & & & & \\
G.HR.M & $175.75 \pm 77.19$ & $143.00 \pm 69.19$ & $32.75 \pm 13.99$ & $0.044^{*}$ \\
G.HT.M & $319.19 \pm 102.82$ & $263.82 \pm 65.53$ & $55.36 \pm 22.34$ & $0.035^{*}$ \\
G.HT.P & $440.49 \pm 185.92$ & $369.31 \pm 169.88$ & $71.17 \pm 19.08$ & $0.005^{*}$ \\
\hline
\end{tabular}

Values are presented as mean \pm standard error.

$\%$ RVC, percentage of the voluntary reference contraction; G, ground gait training group; S, sand ground gait training group; G.HB.M, gait hemiside biceps femoris mean; G.HB.P, gait hemiside biceps femoris peak; G.HR.M, gait hemiside rectus femoris mean; G.HT.M, gait hemiside tibialis anterior mean; G.HT.P, gait hemiside tibialis anterior peak.

${ }^{*} P \leq 0.05$. 


\section{DISCUSSION}

The present study was conducted to examine the effects of sand surface training versus general surface training on improvements to gait ability in chronic stroke patients. Reestablishing gait ability is necessary for patients to return to their communities, and muscle activity changes depending on the training environment. In the TUG tests, which evaluate gait velocity and dynamic stability, both groups showed improved performance times after the intervention, indicating that necessary dynamic stability is enhanced through repetitive gait training and not affected by differences in bearing surfaces.

In the 6MWT, which evaluates gait endurance, the sand surface training group showed significant increases in performance distance, indicating that the training effects of sand surfaces, which are more difficult to walk on than general surfaces, were larger, leading to concomitant improvements in gait ability. Sand surface gait training can increase motility of the lower limb joints and activation of lower limb muscles, leading to neuromuscular involvement in these environments that improved gait ability on general surfaces, resulting in increased gait endurance. These results are consistent with those from a previous study indicating that athletic training on sand surfaces improved strength and endurance in the calf and thigh muscles (Yigit and Tuncel, 1998); another study also indicated that maximum oxygen uptake, heart rate, and ventilatory volume increased through this same type of training (Binnie et al., 2014). Although different patients were tested in the previous studies than in the present study, results suggest that sand surface versus general surface gait training brings about greater improvements in muscle strength, muscle endurance, and cardiopulmonary functions, leading to greater gait endurance.

Reduction in muscle activity on the paretic side leg could be identified from the electromyography data. The relationship between electromyography and improvements in gait ability indicates that as muscle activity decreased, muscle efficiency increased in gaits on general surfaces through training in more difficult environments, leading to measurable functional improvement in the muscles. A previous study reported that lower limb muscle activity was greater on sand surfaces than on general surfaces (Pinnington et al., 2005), and another study indicated that more muscles are mobilized during walking on sand surfaces due to a reduction in surface elastic energy (Zamparo et al., 1992).

A previous study that examined the movements of the ankles of volleyball players during jump training on a sand surface reported that the jumping functions of volleyball players improved during such training as it enabled greater forces to be derived faster from the ankle, because their feet would lose support when trying to push on the sand surface because of its motile characteristics (Impellizzeri et al., 2008). As with these results, increases in the angular speed of the ankle joints and muscle strength through sand surface training could have contributed to improvements in gait ability. As such, when patients returned to the general surfaces with which they were familiar after 6 weeks of training on sand, they could maintain gaits for a longer time with increased muscle strength and endurance.

Along with improvement in the functions of the musculoskeletal system, the improvement of cardiopulmonary functions could have also been a factor in increased gait endurance. A previous study reported that training on sand surfaces improves cardiopulmonary functions such as oxygen uptake (Davies and Mackinnon, 2006); another previous study indicated that sand surface training improves motor functions through high energy consumption and metabolism, as with maximum intensity running, even without reaching maximum speed. Functional threshold values can thereby be improved while still being conscious of injury prevention and rehabilitation programs as it increases the restricted stretching of muscles (Gaudino et al., 2013). Still another previous study reported that muscle damage or injuries were less frequent during training on sand surfaces than during training on general surfaces because of sand's shock absorption and irregularity (Miyama and Nosaka, 2004). Overall, various previous studies indicate that sand surface training leads to functional improvements while reducing fatigue, supporting the results of the present study. Although patients from previous studies were different from the patients of the present study, sand surface training is ideal for gait training in that it improves maximal muscle strength and muscle endurance while limiting muscle damage and injuries.

Although thus far, sand surface training has been utilized mostly for functional improvement training in athletes, this study demonstrated that it can be effective for improvement in musculoskeletal functions of patients with nervous system damage such as stroke patients, and is a suitable therapeutic approach for the postoperative rehabilitation process due to its injury prevention attributes.

Certain limitations of the present study must be acknowledged. First, foot switches were not used to collect lower limb electromyogram data during gaits. Thus gait cycles could not be objectively evaluated. Second, many errors presented in the patient electrograms, and not all electromyographic data could be utilized in analyses. Future studies on the training effects of sand surfaces, 
including more patients and more objective gait analyses, are needed to further elucidate sand surface training mechanics and outcomes.

\section{CONFLICT OF INTEREST}

No potential conflict of interest relevant to this article was reported.

\section{ACKNOWLEDGMENTS}

This research was supported by the Daegu University Research Grant, 2018.

\section{REFERENCES}

Binnie MJ, Dawson B, Arnot MA, Pinnington H, Landers G, Peeling P. Effect of sand versus grass training surfaces during an 8-week pre-season conditioning programme in team sport athletes. J Sports Sci 2014;32: 1001-1012

Bonan IV, Yelnik AP, Colle FM, Michaud C, Normand E, Panigot B, Roth $\mathrm{P}$, Guichard JP, Vicaut E. Reliance on visual information after stroke. Part II: effectiveness of a balance rehabilitation program with visual cue deprivation after stroke: a randomized controlled trial. Arch Phys Med Rehabil 2004;85:274-278.

Caraffa A, Cerulli G, Projetti M, Aisa G, Rizzo A. Prevention of anterior cruciate ligament injuries in soccer. A prospective controlled study of proprioceptive training. Knee Surg Sports Traumatol Arthrosc 1996;4: 19-21.

Carter JM, Beam WC, McMahan SG, Barr ML, Brown LE. The effects of stability ball training on spinal stability in sedentary individuals. J Strength Cond Res 2006;20:429-435.

Davies SE, Mackinnon SN. The energetics of walking on sand and grass at various speeds. Ergonomics 2006;49:651-660.

Franceschini M, Carda S, Agosti M, Antenucci R, Malgrati D, Cisari C; Gruppo Italiano Studio Allevio Carico Ictus. Walking after stroke: what does treadmill training with body weight support add to overground gait training in patients early after stroke?: a single-blind, randomized, controlled trial. Stroke 2009;40:3079-3085.

Gaudino P, Gaudino C, Alberti G, Minetti AE. Biomechanics and predicted energetics of sprinting on sand: hints for soccer training. J Sci Med Sport 2013;16:271-275

Impellizzeri FM, Rampinini E, Castagna C, Martino F, Fiorini S, Wisloff U. Effect of plyometric training on sand versus grass on muscle soreness and jumping and sprinting ability in soccer players. Br J Sports Med
2008;42:42-46.

Lord S, McPherson KM, McNaughton HK, Rochester L, Weatherall M. How feasible is the attainment of community ambulation after stroke? A pilot randomized controlled trial to evaluate community-based physiotherapy in subacute stroke. Clin Rehabil 2008;22:215-225.

Maynard V, Bakheit AM, Shaw S. Comparison of the impact of a single session of isokinetic or isotonic muscle stretch on gait in patients with spastic hemiparesis. Clin Rehabil 2005;19:146-154.

Miyama M, Nosaka K. Influence of surface on muscle damage and soreness induced by consecutive drop jumps. J Strength Cond Res 2004; 18:206-211

Mossberg KA, Fortini E. Responsiveness and validity of the six-minute walk test in individuals with traumatic brain injury. Phys Ther 2012; 92:726-733.

Neumann DA. Kinesiology of the musculoskeletal system: foundations for rehabilitation. London: Elsevier Health Sciences; 2013.

Park YH, Kim YM, Lee BH. An ankle proprioceptive control program improves balance, gait ability of chronic stroke patients. J Phys Ther Sci 2013;25:1321-1324

Patla AE, Shumway-Cook A. Dimensions of mobility: defining the complexity and difficulty associated with community mobility. J Aging Phys Act 1999;7:7-19.

Pinnington HC, Lloyd DG, Besier TF, Dawson B. Kinematic and electromyography analysis of submaximal differences running on a firm surface compared with soft, dry sand. Eur J Appl Physiol 2005;94:242253.

Podsiadlo D, Richardson S. The timed "Up \& Go": a test of basic functional mobility for frail elderly persons. J Am Geriatr Soc 1991;39:142-148.

Raju RS, Sarma PS, Pandian JD. Psychosocial problems, quality of life, and functional independence among Indian stroke survivors. Stroke 2010; 41:2932-2937.

Schmid AA, Van Puymbroeck M, Altenburger PA, Dierks TA, Miller KK, Damush TM, Williams LS. Balance and balance self-efficacy are associated with activity and participation after stroke: a cross-sectional study in people with chronic stroke. Arch Phys Med Rehabil 2012;93: 1101-1107.

Schulthies SS, Ricard MD, Alexander KJ, Myrer JW. An electromyographic investigation of 4 elastic-tubing closed kinetic chain exercises after anterior cruciate ligament reconstruction. J Athl Train 1998;33:328-335.

Shumway-Cook A, Patla AE, Stewart A, Ferrucci L, Ciol MA, Guralnik JM. Environmental demands associated with community mobility in older adults with and without mobility disabilities. Phys Ther 2002;82: 670-681.

Yigit SS, Tuncel F. A comparison of the endurance training responses to road and sand running in high school and college students. J Strength 
Cond Res 1998;12:79-81.

Zamparo P, Perini R, Orizio C, Sacher M, Ferretti G. The energy cost of walking or running on sand. Eur J Appl Physiol Occup Physiol 1992; 65:183-187. 